\title{
Temporary bilateral sensorineural hearing loss following cardiopulmonary bypass
}

\section{-A case report-}

\author{
Hyo Jung Son, Jung Hwa Joh, Wook Jong Kim, Ji Hyun Chin, Dae Kee Choi, Eun Ho Lee, Ji Yeon Sim, \\ and In-Cheol Choi
}

Department of Anesthesiology and Pain Medicine, Asan Medical Center, University of Ulsan College of Medicine, Seoul, Korea

Sudden sensorineural hearing loss has been reported to occur following anesthesia and various non-otologic surgeries, mostly after procedures involving cardiopulmonary bypass. Unilateral sensorineural hearing loss resulting from microembolism is an infrequent complication of cardiopulmonary bypass surgery that has long been acknowledged. Moreover, there are few reports on the occurrence of bilateral sensorineural hearing loss without other neurologic deficits and its etiology has also not been determined. We describe here a rare case of bilateral hearing loss without other neurologic deficits in an otherwise healthy 27-year-old woman who underwent cardiopulmonary bypass surgery for repair of severe mitral valve stenosis. The patient suffered from profound sensorineural hearing loss in both ears that was recognized immediately upon extubation, and audiometry tests confirmed the diagnosis. Without any treatment, her hearing recovered almost completely by the time of her discharge one week after surgery. (Korean J Anesthesiol 2011; 61: 162-165)

Key Words: Cardiopulmonary bypass, Hearing loss, Postoperative complication.

Sudden sensorineural hearing loss (SNHL), a manifestation of cochlear injury, is defined as a hearing loss of more than $30 \mathrm{~dB}$ in at least three contiguous audiometric frequencies developing over a period of a few hours to 3 days [1]. SNHL has been estimated to affect approximately 1 of 5,000 people in the general population [2]. In most cases, its exact cause is not determined, although various etiologies proposed to explain the phenomenon include vascular mechanisms (microembolism, hemorrhage, thrombosis), inflammatory, autoimmune, metabolic, traumatic, and idiopathic mechanisms.

SNHL is also an uncommon complication of cardiopulmonary bypass (CPB). We describe here, a patient who experienced bilateral SNHL immediately upon emergence from anesthesia after undergoing thoracoscopic mitral valvuloplasty under CPB.

Received: October 13, 2010. Revised: 1st, November 17, 2010; 2nd, January 3, 2011; 3rd, February 1, 2011; 4th, February 15, 2011. Accepted: February 18, 2011.

Corresponding author: In-Cheol Choi, M.D., Department of Anesthesiology and Pain Medicine, Asan Medical Center, University of Ulsan College of Medicine, 388-1, Pungnap-2dong, Songpa-gu, Seoul 138-736, Korea. Tel: 82-2-3010-3862, Fax: 82-2-470-1363, E-mail: icchoi@amc.seoul.kr (ㄷ) This is an open-access article distributed under the terms of the Creative Commons Attribution Non-Commercial License (http:// creativecommons.org/licenses/by-nc/3.0/), which permits unrestricted non-commercial use, distribution, and reproduction in any medium, provided the original work is properly cited. 


\section{Case Report}

A 27-year-old female patient who had developed progressive dyspnea and chest discomfort after spontaneous delivery 2 months ago was diagnosed with severe mitral stenosis (MS) accompanied by mitral regurgitation (MR). Her height and weight were $154.6 \mathrm{~cm}$ and $51.1 \mathrm{~kg}$, respectively. She was scheduled for mitral valvuloplasty under minimally invasive thoracic incision using Automated Endoscopic System for Optimal Positing (AESOP, Computer Motion, Santa Barbara, CA, USA). Preoperative evaluation showed nothing abnormal except for severe MS. Her recent medication history included ramipril and torasemide for symptomatic relief of dyspnea.

Prior to induction of anesthesia, a 5-lead electrograph, a non-invasive hemodynamometer, a pulse oximeter, a BIS sensor, and two cerebral oxymetry sensors were placed and monitored. After sufficient denitrogenation with $100 \%$ oxygen, anesthesia was induced with $12 \mathrm{mg}$ of etomidate, $50 \mathrm{mg}$ of rocuronium, and continuous infusion of propofol (target effect site concentration $1.0 \mathrm{ug} / \mathrm{ml}$ ) and remifentanil (target effect site concentration $10 \mathrm{ng} / \mathrm{ml}$ ) using a target controlled infusion system (Orchestra $^{\circledR}$, Fresenius vial, Brezims, France). After intra-arterial cannulation of the left radial artery, endotracheal intubation was performed uneventfully. A central venous catheter MAC $^{\mathrm{TM}}$ Two-Lumen Central Venous Access set, ARROW $^{\circledR}$, Reading, PA, USA) with a Swan-Ganz catheter (Swan-Ganz CCOmbo, CCO/SvO2/CEDV, Edwards lifesciences LLC, Irvine, CA, USA) was placed in her right subclavian vein.
Superior vena cava (SVC) cannulation was performed with a 17 Fr femoral arterial cannula $\left(D_{L P}{ }^{\circledR}\right.$, Medtronic inc.,Minneapolis, MN, USA) in her right internal jugular vein after injection of 1530 units of heparin. During the operation, anesthesia was maintained with oxygen $(2 \mathrm{~L} / \mathrm{min})$ and continuous infusion of propofol (target effect site concentration 1.0-1.5 ug/ml), remifentanil (target effect site concentration $10-20 \mathrm{ng} / \mathrm{ml}$ ), and rocuronium $5 \mathrm{ug} / \mathrm{kg} / \mathrm{min}$. There were no clinically significant periods of hypotension during induction and maintenance of anesthesia. There were also no adverse events during CPB, and perfusion pressures were well-maintained at 50-78 mmHg. After initial mitral valvuloplasty and CPB weaning, intraoperative transesophageal echocardiography (TEE) was performed, which revealed mild to moderate degree of MR. $\mathrm{CPB}$ was restarted and valvuloplasty was performed again. The second attempt was successful without signs of regurgitation or stenosis. Total CPB time was 101 minutes, pump flow rate was maintained at an appropriate level of $2.4 \mathrm{~L} / \mathrm{min} / \mathrm{m}^{2}$ and mean arterial pressure was maintained over $50 \mathrm{mmHg}$. Monitoring of cerebral blood flow adequacy by brain oxymeters showed no significant decreases in cerebral blood flow during CPB. Lowest temperature under $\mathrm{CPB}$, as measured by an esophageal stethoscope, was $27.3^{\circ} \mathrm{C}$. The patient was transferred to the intensive care unit after surgery and was extubated 5 hours later. Immediately after extubation, the patient complained of profound bilateral hearing loss. Bilateral ear fullness and tinnitus were present, but not vertigo. Otoscopic examination on the same day showed that both ears were normal and there

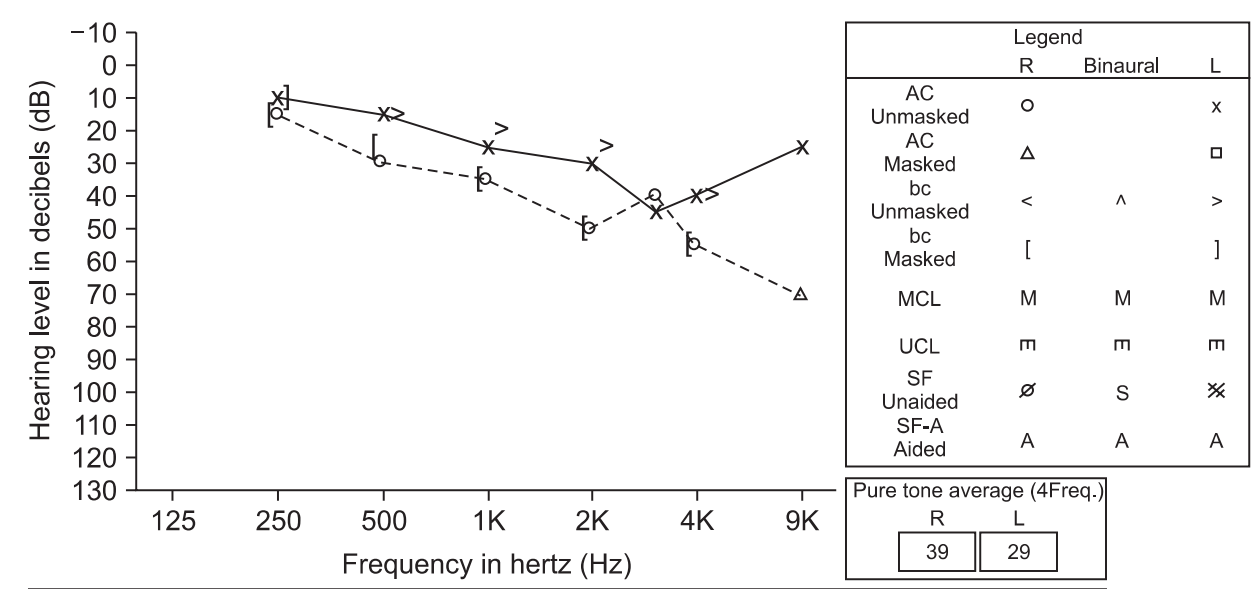

\begin{tabular}{|c|c|c|c|c|c|c|c|c|c|c|c|}
\hline & \multicolumn{4}{|c|}{ Speech audiometry } & & \multicolumn{6}{|c|}{ Word recognition } \\
\hline & SRT & Mask & $\mathrm{MCL}$ & UCL & & $\%$ & Stimulus & Mask & $\%$ & Stimulus & Noise \\
\hline $\mathrm{R}$ & 28 & & & & $\mathrm{R}$ & 92 & 68 & & & & \\
\hline $\mathrm{L}$ & 12 & & & & L & 96 & 52 & & & & \\
\hline Binaural & & & & & Binaural & & & & & & \\
\hline $\mathrm{SF}$ & & & & & SF & & & & & & \\
\hline SF-A & & & & & SF-A & & & & & & \\
\hline SF-A2 & & & & & SF-A2 & & & & & & \\
\hline
\end{tabular}

Fig. 1. Pure tone audiometry and speech audiometry performed on postoperative day 2 is consistent with profound bilateral sensorineural hearing loss. The right ear was more affected, and predominated in the high frequency range. AC: air conduction, bc: bone conduction, SRT: speech reception threshold. 


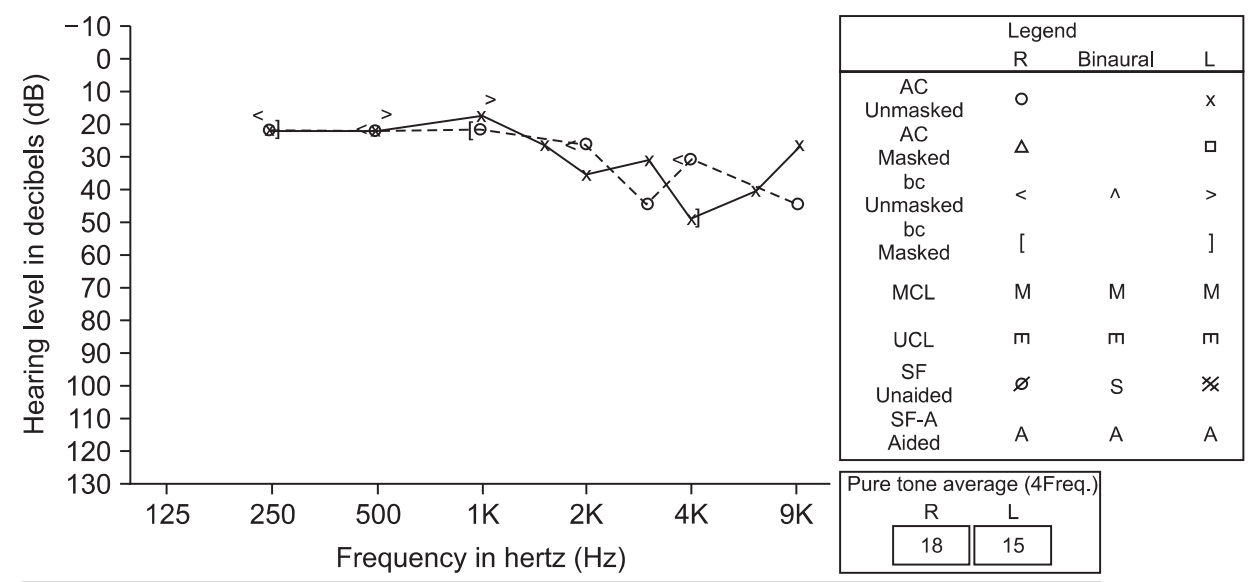

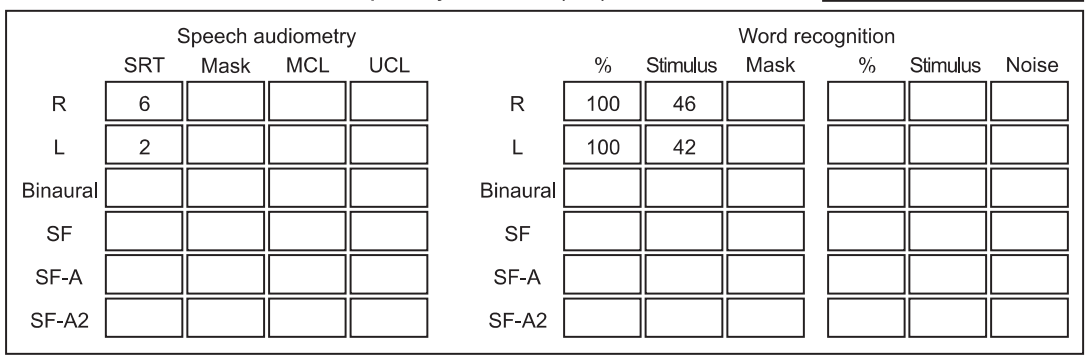

were no other neurologic abnormalities. On postoperative day 1 the patient's hearing improved slightly. Audiometry results on postoperative day 2 (Fig. 1) indicated acute SNHL with hearing impairment in the high frequency range, with the right ear more affected. Due to the immediate postoperative state of the patient, high dose steroid therapy was not initiated and intratympanic steroid injection was considered if she did not recover. However, without any therapy, the patient's hearing had recovered such that she did not feel very uncomfortable by postoperative day 3. Follow up audiometry on postoperative day 4 also demonstrated improved hearing in both ears. The patient was discharged one week after surgery, and postoperative echocardiography demonstrated successful mitral valve repair without dehiscence or pathologic regurgitation. Additional audiometry on postoperative day 17 (Fig. 2) showed that her hearing had almost fully recovered. By this time the patient had no complaints of hearing impairment, and no further follow up of her hearing was performed.

\section{Discussion}

SNHL may be a complication of otologic surgeries, but has also been known to occur after non-otologic surgeries under general anesthesia $[3,4]$. Though it has rarely been documented to occur after surgeries which do not involve CPB [5], most cases of SNHL following general anesthesia for non-otologic surgeries involve the use of CPB for cardiac surgeries [6-8]. A larger retrospective study in 1981 found that unilateral SNHL immediately after CPB occurred in 7 to 7,000 patients $(0.1 \%)$, and none of whom show ed full recovery [9]. Although there is a lack of review articles on this subject since 1981, the onset and the severity of hearing impairment and the extent of recovery have been found to vary in summary of the case reports published since 1972 [6-8,10].

As in our patient, the etiology of SNHL after CPB has not yet been established. Microembolisms generated in the pump oxygenator systems with occlusion of the cochlear branch of the internal auditory artery are considered the most likely cause of SNHL following cardiovascular surgery $[6,7,9,10]$. It has been typically associated with unilateral hearing loss, and rarely been considered a cause of bilateral hearing loss. Sources of emboli may be gas bubbles or biological aggregates including thrombus, lipids, platelet aggregates and particulate matter from calcified vessels or valves [6]. Microembolisms in the inner ear can cause ischemia of the stria vascularis and hair cells, which may result in irreversible hearing impairment [11]. Hemodynamic fluctuations such as prolonged hypotension during CPB has been advocated as a cause of SNHL following CPB [8]. In the case of our patient, her vital signs were stable throughout the operation, and there were no prolonged periods of hypotension except for the brief period of hypotension immediately post-CPB period. Moreover, hypoperfusion due to hypotension is not likely to cause of SNHL without other abnormalities, as hypoperfusion would also have affected other major organs susceptible to ischemic insults, including the brain, heart, kidneys and intestines. Hypothermia during 
CPB also can cause SNHL, however, it also have affected other major organs vulnerable to hypothermic insult. Ototoxicity may account for hearing loss in general practice as well as after CPB. Drugs frequently associated with ototoxicity include aminoglycosides, erythromycin, vancomycin, loop diuretics, salicylates, nonsteroidal anti-inflammatory agents, and antineoplastic drugs [11]. Our patient, however, did not receive any potentially ototoxic drugs in concentrations that might cause ototoxicity. Although hypercoagulable states potentiating thromboembolism may also cause SNHL, our patient did not receive blood products (platelet concentrates or fresh frozen plasma) or drugs such as tranexamic acid or aprotinin that could result in produce hypercoagulability, and postoperative laboratory findings in the intensive care unit showed that her coagulation state was normal. SNHL may also be caused by injury to the central nervous system, but as our patient did not show any other abnormal neurological findings, this was very unlikely in our patient. Nitrous oxide can provoke SNHL by increasing middle ear pressure and creating membrane breaks and perilymphatic fistulas $[7,12]$. Considering all of these possible causes, although with a very small possibility, occlusion of both internal auditory arteries by microthrombi cannot be ruled out for the SNHL that developed in our patient. A higher prevalence of mitral valve prolapse and mitral regurgitation has been observed in patients with idiopathic SNHL, suggesting that the patient's disease or physical status may be responsible for at least some cases of SNHL [13]. In addition, the SNHL occurring after CPB in our patient may be coincidental, not unlike idiopathic sudden hearing loss in the general population.

The mainstay of treatment, if any, is usually based on corticosteroid administration regardless of the initiating cause of SNHL. Patients with hearing impairment after CPB have been shown to have a poorer prognosis than patients who experience hearing loss after other procedures, with less than $50 \%$ of the former showing recovery $[2,6,7,10,11]$. The initial shape of the audiogram has been found to have significant correlation with the extent of recovery [14]. Hearing impairment in the high frequency range is considered an unfavorable factor, whereas a low to middle frequency audiogram contour is associated with an increased likehood of recovery. SNHL following CPB has been reported to occur mainly in the high frequency range $[12,15]$. Audiometry showed that the hearing loss in our patient was confirmed by audiometry to be mainly in the high frequency range. However, she showed signs of recovery beginning the day after CPB and fully recovered without steroid treatment.

In conclusion, SNHL may occur after various anesthetic procedures, and those following surgeries using CPB are of particular concern. Although there have been many case reports and efforts have been made to estimate the overall incidence of SNHL after CPB, transient subclinical forms of SNHL likely go unnoticed since most patients do not undergo pre-operative and post-operative audiometry tests. Thus the exact incidence of SNHL after CPB remains unknown, but may be higher than is generally assumed. Anesthesiologist should have a better understanding of the incidence and prognosis of hearing impairment after $\mathrm{CPB}$, as well as its potential causes, thus minimizing the risk of SNHL after CPB.

\section{References}

1. Koç A, Sanisoğlu O. Sudden sensorineural hearing loss: literature survey on recent studies. J Otolaryngol 2003; 32: 308-13.

2. Byl FM Jr. Sudden hearing loss: eight years' experience and suggested prognostic table. Laryngoscope 1984; 94: 647-61.

3. Evan KE, Tavill MA, Goldberg AN, Silverstein H. Sudden sensorineural hearing loss after general anesthesia for nonotologic surgery. Laryngoscope 1997; 107: 747-52.

4. de la Cruz M, Bance M. Bilateral sudden sensorineural hearing loss following non-otologic surgery. J Laryngol Otol 1998; 112: 769-71.

5. Cox AJ 3rd, Sargent EW. Sudden sensorineural hearing loss following nonotologic, noncardiopulmonary bypass surgery. Arch Otolaryngol Head Neck Surg 1997; 123: 994-8.

6. Arenberg IK, Allen GW, Deboer A. Sudden deafness immediately following cardiopulmonary bypass. J Laryngol Otol 1972; 86: 73-7.

7. Wright JL, Saunders SH. Sudden deafness following cardiopulmonary bypass surgery. J Laryngol Otol 1975; 89: 757-9.

8. Shapiro MJ, Purn JM, Raskin C. A study of the effects of cardiopulmonary bypass surgery on auditory function. Laryngoscope 1981; 91: 2046-52.

9. Plasse HM, Mittleman M, Frost JO. Unilateral sudden hearing loss after open heart surgery: a detailed study of seven cases. Laryngoscope 1981; 91: 101-9.

10. Walsted A, Andreassen UK, Berthelsen PG, Olesen A. Hearing loss after cardiopulmonary bypass surgery. Eur Arch Otorhinolaryngol 2000; 257: 124-7.

11. Sprung J, Bourke DL, Contreras MG, Warner ME, Findlay J. Perioperative hearing impairment. Anesthesiology 2003; 98: 241-57.

12. Iriz A, Cagli K, Gocer C, Dursun E, Korkmaz H, Eryilmaz A. Effects of open heart surgery on hearing thresholds measured by high frequency audiometry. J Laryngol Otol 2008; 122: 795-8.

13. Vazquez R, Solanellas J, Alfageme I, Valenzuela-Garcia LF, Pavon R, Leal J, et al. Mitral valve prolapse and sudden deafness. Int J Cardiol 2008; 124: 370-1.

14. Xenellis J, Karapatsas I, Papadimitriou N, Nikolopoulos T, Maragoudakis $\mathrm{P}$, Tzagkaroulakis $\mathrm{M}$, et al. Idiopathic sudden sensorineural hearing loss: prognostic factors. J Laryngol Otol 2006; 120: 718-24.

15. Phillipps JJ, Thornton AR. Audiometric changes in patients undergoing coronary artery bypass surgery. Br J Audiol 1996; 30: 19-25. 